\title{
Pruebas de función plaquetaria por el sistema PFA-200
}

\author{
PFA-200 system for platelet dysfunction analysis
}

\author{
Laura Rivillas-Rigol', Jennifer C. Vizcaíno-Carruyo², Verónica J. Tangarife- \\ Castaño3 ${ }^{3}$ Germán Campuzano-Zuluaga ${ }^{4}$, Ana Isabel Toro-Montoya ${ }^{5}$
}

\section{Utilidad clínica de la prueba}

Las pruebas de función plaquetaria realizadas con el analizador PFA-200 (del inglés, Platelet Function Analyzer) han venido reemplazando el tiempo de sangría, una prueba ya obsoleta, de acuerdo con el College of American Pathologists (CAP) y la American Society for Clinical Pathology (ASCP). Este analizador, mediante un sistema que utiliza cartuchos, posee la capacidad de simular in vitro las condiciones hemodinámicas del paciente para el tamizaje rápido, sencillo y automatizado de la disfunción plaquetaria. Las pruebas son de utilidad para evaluar la función plaquetaria en pacientes con alteraciones como enfermedad de von Willebrand y otras trombocitopatías congénitas o adquiridas, y evalúa la disfunción plaquetaria causada por inhibidores de la agregación plaquetaria, como la aspirina. Además, son de gran utilidad para el tamizaje prequirúrgico y en los embarazos de alto riego. Este sistema ofrece con su más reciente prueba, la INNOVANCE ${ }^{\oplus}$ PA-200 P2Y, la posibilidad de evaluar la respuesta de pacientes que están siendo tratados con antagonistas del receptor plaquetario P2Y12, como son clopidogrel, prasugrel y ticagrelor, determinando el porcentaje de adhesión y agregación plaquetaria, logrando discriminar entre un paciente antiagregado y un paciente resistente al tratamiento.

\footnotetext{
${ }^{1}$ Microbióloga y Bioanalista. Estudiante, MSc en Microbiología y Bioanálisis con énfasis en Coagulación. Bacterióloga de Hemostasia, Laboratorio Clínico Hematológico S.A. Medellín, Colombia.

${ }^{2}$ Médica, Especialista en Hematología. Asistente Científica, Editora Médica Colombiana S.A. Medellín, Colombia.

${ }_{3}^{3}$ Microbióloga y Bioanalista. MSc en Ciencias Básicas Biomédicas. Coordinadora de Unidad de Investigación, Desarrollo y Academia. Laboratorio Clínico Hematológico S.A.-Editora Médica Colombiana S.A. Medellín, Colombia.

${ }^{4}$ Médico, Especialista en Patología Anatómica y Clínica, Subespecialista en Hematopatología. Fellow, College of American Pathologists (FCAP). Director, Laboratorio Clínico Hematológico S.A. Director, Editora Médica Colombiana S.A. Medellín, Colombia.

${ }^{5}$ Bacterióloga y Laboratorista Clínica. MSc en Virología. Directora Científica, Editora Médica Colombiana S.A. Medellín,

Colombia. E-mail: atoro@edimeco.com.
} 


\section{Fundamento de la técnica}

El sistema PFA-200 está diseñado para la medición in vitro de la función plaquetaria en sangre total anticoagulada. El sistema incluye una serie de cartuchos, conformados por un capilar, un depósito para la muestra y una membrana de nitrocelulosa con actividad bioquímica.

Para la prueba, la sangre es depositada en el orificio del cartucho, para luego ser aspirada a través del capilar. Gracias a una gran fuerza de cizallamiento generada por la presión negativa del instrumento sobre el cartucho, las plaquetas en la muestra entran en contacto con la membrana impregnada con una solución activadora, disolviendo los reactivos que la recubren; estos proporcionan estimulación controlada a las plaquetas, a medida que la muestra de sangre pasa a través de la abertura. Durante la prueba, las plaquetas se adhieren a la membrana, se activan y liberan su contenido granular, iniciándose su agregación hasta producir la oclusión del capilar, lo cual finalmente detiene el flujo de sangre.

El tiempo transcurrido desde el inicio de la prueba hasta la oclusión de la abertura es determinado y registrado por un microprocesador en intervalos de tiempo, generando el resultado como un Tiempo de Obturación (TO) en segundos, siendo este tiempo el indicador de la función plaquetaria de la muestra analizada.

\section{Preparación del paciente}

Aunque no se requiere preparación especial, en lo posible el paciente debe estar en ayunas. Se recomienda evitar dietas ricas en grasa en las 8 a
12 horas previas a la toma de la muestra y evitar el ejercicio físico intenso 24 horas antes. Adicionalmente, se debe evitar el consumo de alcohol y cigarrillo previo a la toma de la muestra y permanecer en reposo durante 10 a 15 minutos antes de la venopunción. Debido al ciclo circadiano, las muestras se deben extraer entre las 6:00 a.m. y las 10:00 a.m.

En los pacientes que reciben tratamiento, es importante que haya una adecuada adherencia al mismo, durante al menos los 7 días previos a la toma de la muestra. Ante la presencia de un resultado que indique resistencia a los medicamentos, el médico tratante debe hacer una evaluación rigurosa para descartar una falla en la adherencia.

\section{Muestra}

Sangre total, en tubo con citrato de sodio al 3,2\% (tapa azul celeste), utilizando aguja calibre $21 \mathrm{G}$ (aguja verde) o superior. El tubo con citrato debe ser el primero en orden de extracción; se debe llenar hasta agotar el vacío y mezclar inmediatamente por inversión. El torniquete se debe aplicar máximo durante un minuto. La muestra no se debe extraer de catéter. Si durante la toma de la muestra se produce un colapso venoso o el flujo de sangre se interrumpe, se debe repetir la toma de la muestra en otra vena.

\section{Almacenamiento y preservación de las muestras}

La muestra no debe ser centrifugada, ya que se activan las plaquetas. Debe permanecer en reposo y a temperatura ambiente $\left(15^{\circ} \mathrm{C}\right.$ a $\left.25^{\circ} \mathrm{C}\right)$ durante mínimo 10 minutos, y se debe procesar antes de 4 horas. 


\section{Interpretación de los resultados}

Es importante que el médico que ordena las pruebas tenga claridad al momento de hacer el requerimiento; si desea una valoración de la función plaquetaria debe solicitar una prueba que mida el Tiempo de cierre con colágeno/epinefrina y el Tiempo de cierre con colágeno/ADP, en tanto que si requiere la prueba para el monitoreo del tratamiento con antagonistas del P2Y12, debe solicitar específicamente la prueba P2Y12. Los valores de referencia tienen poca variación entre los laboratorios; sin embargo, deben ser estandarizados en cada institución. En el Laboratorio Clínico Hematológico de Medellín (Colombia), para la prueba que se asocia con una posible disfunción plaquetaria por defectos intrínsecos de las plaquetas, enfermedad de von Willebrand o presencia de agentes inhibidores plaquetarios, como la aspirina, los valores de referencia son: Tiempo de cierre con colágeno/epinefrina entre 80 y 150 segundos, y Tiempo de cierre con colágeno/ADP entre 60 y 100 segundos. Cuando se utiliza la prueba INNOVANCE ${ }^{\oplus}$ PFA-200 P2Y, el patrón típico en pacientes con respuesta adecuada al tratamiento con antagonistas del P2Y12, es un tiempo de obturación prolongado; es decir, de más de 106 segundos.

\section{Interferentes y limitaciones}

Algunos medicamentos como los antiinflamatorios no esteroideos y antibióticos pueden interferir con los resultados de la prueba. De igual forma, alimentos, sustancias y medicamentos naturistas como el jengibre, Ginkgo bi- loba, omega-3 y 6, castaño de indias, té verde y marihuana, y los arándanos, entre otros, también pueden afectar los resultados. En general, se recomienda la suspensión de estos interferentes durante la semana previa a la realización de la prueba.

En cuanto a la muestra, no se deben utilizar muestras de sangre hemolizadas ni con coágulos, debido a que pueden producir resultados prolongados u obstrucciones del flujo en el cartucho. Valores de hematocrito y recuento de plaquetas anormalmente disminuidos pueden producir prolongación del tiempo de obturación, al igual que una concentración alta de lípidos en la muestra.

\section{Referencias}

1. Siemens Healthineers. INNOVANCE PFA-200* Function Description System. Functional description; 2011. Acceso 17 de febrero de 2020. Disponible en https://www.siemens-healthineers.com/co/hemostasis/systems/innovancepfa-200\#FEATURES_BENEFITS.

2. Siemens Healthcare. Inserto INNOVANCE PFA P2Y. Referencia B4170G22C0504 (1210), mayo 2012.

3. Lim HH, Li S, An GD, Woo KS, Kim KH, Kim JM, et al. Platelet Function Analyzer-200 P2Y results are predictive of the risk of major adverse cardiac events in Korean patients receiving clopidogrel therapy following acute coronary syndrome. Ann Lab Med 2018;38:413-419. https://doi. org/10.3343/alm.2018.38.5.413.

4. Linnemann B, Schwonberg J, Rechner AR, Mani H, Lindhoff-Last E. Assessment of clopidogrel non-response by the PFA-100 system using the new test cartridge INNOVANCE PFA P2Y. Ann Hematol 2010;89:597-605. https://doi.org/10.1007/ s00277-009-0881-9.

5. Campuzano-Maya G. PFA-100: una nueva prueba de función plaquetaria sustituta del tiempo de sangría. Med Lab 2013;19:511-548. 